\title{
Tecnologia Industrial Básica (TIB) no Setor Metalmecânico: um Estudo Exploratório no Rio de Janeiro
}

\author{
Heitor Soares Mendes (CEFET/RJ) ${ }^{1}$ \\ Marta Lucia Azevedo Ferreira (CEFET/RJ)²
}

\begin{abstract}
RESUMO
Este artigo se propõe a analisar a capacitação em Tecnologia Industrial Básica (TIB) de Micro, Pequenas e Médias Empresas (MPME) do setor metalmecânico localizadas na Zona Oeste (ZO) do município do Rio de Janeiro (MRJ) no âmbito do Sistema Brasileiro de Tecnologia (Sibratec) e de uma de suas redes regionais de extensão tecnológica - Sibratec-ET Rede RJ. A demanda das empresas é confrontada com esta oferta de apoio em atividades de normalização e regulamentação técnica, metrologia, bem como de avaliação de conformidade e qualidade. Trata-se de uma pesquisa qualitativa, empírica e exploratória baseada no método do estudo de caso. Confirmando a literatura, os resultados mostram a fragilidade da maioria das empresas quanto à capacitação em TIB. A análise da demanda vis-à-vis a oferta de apoio revelou uma correlação positiva favorecida pelo formato flexível do atendimento proposto. Ainda assim, a Rede Sibratec-ET Rede RJ não logrou êxito em relação a seus objetivos, configurando-se mais como um arranjo institucional potencialmente positivo do que efetivo. Ademais, as empresas pesquisadas sequer souberam da existência da Sibratec-ET ou foram visitadas o que indica um campo fértil para o debate a partir de uma avaliação de impacto deste arranjo específico em outros setores ou regiões ou mesmo do Sibratec como um todo. Ratifica-se a necessidade de novas pesquisas sobre esses temas, sobretudo considerando a dimensão nacional do Sibratec e sua forma de atuação em distintos tipos de redes como centros de inovação, serviços tecnológicos e a de extensão tecnológica aqui abordada.
\end{abstract}

PALAVRA-CHAVE: Tecnologia Industrial Básica (TIB). Micro, Pequenas e Médias Empresas (MPME). Setor Metalmecânico. Extensão Tecnológica. Sibratec-ET Rede-RJ.

\section{ABSTRACT}

This paper analyzes the Infrastructural Technologies (IT) of the Micro, Small and Medium Enterprises (MSME) of the metalworking sector in the Western Region of the Rio de Janeiro state in the context of the Brazilian System of Technology (Sibratec) and of the one of its regional technological extension networks called Sibratec-ET Rede RJ. The demand of companies is confronted with this offer of support in activities of standardization and technical regulation, metrology, as well as conformity and quality assessment. This is a qualitative, empirical and exploratory research based on the case study method. Confirming the literature, the results show the fragility of most companies regarding TIB competences. The analysis of the demands of the MSME vis-à-vis the offer of support from Sibratec Technological Extension revealed a positive correlation, favoured by the flexible format of this network. Futhermore, the Sibratec-RJ network was not successful in relation to its objectives, representing more like a potentially positive institutional arrangement than effective. In addition, the enterprises surveyed have taken neither notice of the existence of Sibratec-RJ, nor they have been visited by extension agents, which indicates a fertile field for debate from an impact assessment of this specific arrangement in other sectors or regions or even Sibratec as one all. The need for new research on these themes is confirmed, especially considering the national dimension of the system and its way of acting in different types of networks such as innovation centers, technological services and technological extension.

KEYWORDS: Infrastructural Technology Capability. Micro, Small and Medium-sized Enterprises (MSME). Metalworking Sector. Technological Extension. Sibratec-ET Rede RJ.

1 Doutor em Ciências em Políticas Públicas, Estratégias e Desenvolvimento (IE/UFRJ), é docente e pesquisador do CEFET/RJ e coordenador substituto do Núcleo de Inovação Tecnológica (NIT CEFET/RJ).

2 Doutora em Ciências em Políticas Públicas, Estratégias e Desenvolvimento (IE/UFRJ), é docente e pesquisadora do CEFET/RJ vinculada ao Programa de Pós-Graduação em Desenvolvimento Regional e Sistemas Produtivos (PPDSP) e coordenadora do Núcleo de Inovação Tecnológica (NIT CEFET/RJ). 


\section{1- Introdução}

Este artigo destaca a importância da capacidade tecnológica como base das atividades de produção e inovação das empresas. Esta, por sua vez, é entendida como um processo sistêmico e complexo que possui dimensões políticas, econômicas e sociais (BITTENCOURT \& CARIO, 2017). A capacidade tecnológica é um conjunto ou estoque de recursos à disposição das empresas que depende de aprendizagem tecnológica, ou seja, de vários mecanismos de captação de diferentes tipos de conhecimento tecnológico a partir de fontes internas e externas (FIGUEIREDO, 2012). O modo e a velocidade com que as empresas constroem e acumulam capacidade tecnológica é crucial para a inovação, tendo impactos significativos na sua competitividade.

Tigre (2014) acrescenta que as fontes internas incluem, tanto as atividades voltadas para o desenvolvimento de produtos, processos e métodos de gestão, como aquelas dirigidas à obtenção de melhorias incrementais por meio de treinamentos e programas de qualidade, produtividade e aprendizagem organizacional. As fontes externas englobam operações de busca e aquisição de conhecimentos técnicos em geral codificados, bem como os de natureza tácita que advêm da contratação de especialistas e consultores, do relacionamento com fornecedores e clientes e de estágios e treinamentos. Elas abrangem ainda os contratos de transferência de tecnologia envolvendo patentes e licenças de fabricação, bem como a compra de tecnologias embutidas em máquinas, equipamentos e softwares.

Como afirmam Britto (2017) e Tironi (2017), as empresas raramente inovam sozinhas, pois à medida que se tornam mais especializadas, focalizam suas competências e se articulam com outros agentes econômicos para ter acesso a conhecimentos e know-how complementar. Por isso a cooperação vem se tornando cada vez mais fundamental como forma de intercâmbio de informações e de integração de competências produtivas, tecnológicas e organizacionais. Tratase de uma opção de importância crescente para as empresas que buscam desenvolver vantagens competitivas melhorando suas capacidades tecnológicas, bem como seus produtos, serviços e processos produtivos.

O Estado tem papel-chave no sentido de promover a expansão da infraestrutura científica e tecnológica necessária para que empresas de portes e setores produtivos diversos possam desenvolver tecnologias aplicadas. Ela inclui o sistema público de pesquisa, ou seja, universidades, institutos e laboratórios de pesquisa, além de organizações com poder legal e normativo encarregadas de dar suporte às atividades produtivas agrupadas sob a denominação de Tecnologia Industrial Básica (TIB).

A TIB compreende, não apenas ativos físicos, mas sobretudo técnicas, procedimentos e metodologias de codificação, análise e normalização de produtos e processos. Ela tem como funções básicas normalização, regulamentação técnica, metrologia e avaliação de conformidade, bem como aquelas voltadas para tecnologias de gestão com ênfase em qualidade, propriedade intelectual e informações tecnológicas (TIGRE, 2014). Trata-se de um sistema multidimensional que visa garantir às empresas e aos consumidores a qualidade de produtos e serviços em diferentes mercados. Assim, a TIB envolve um conjunto de funções encadeadas relativas a normas, medidas, ensaios e qualidade, sendo consideradas tecnologias infraestruturais (FLEURY, 2007).

Como mostram Figueiredo (2012), La Rovere et al. (2014) e Mendes et al. (2017), a elevação do padrão tecnológico é difícil para as empresas brasileiras, especialmente para aquelas de menor porte, o que indica a necessidade de incentivos governamentais específicos. Mendes et al. (2017) lembram que as preocupações com o adensamento da estrutura produtiva e do sistema brasileiro de inovação se fortaleceram a partir do século XXI. O Programa de Aceleração do Crescimento (PAC) surgiu em 2007, ao qual se articulou o Plano de Ação em Ciência, Tecnologia e Inovação para o Desenvolvimento Nacional 2007-2010 (PACTI 2007-2010) Foi no âmbito deste Plano que surgiu também em 2007 o Sistema Brasileiro de Tecnologia (Sibratec) visando o apoio ao desenvolvimento tecnológico e inovativo das empresas brasileiras por meio de três tipos de 
redes - centros de inovação, serviços tecnológicos e extensão tecnológica - sendo para esta última dirigida a ênfase deste artigo (BRASIL, 2007).

Como assinalam os autores, um dos aspectos centrais da extensão tecnológica - chamada anteriormente de extensão industrial - é o apoio à modernização das empresas por intermédio da difusão de tecnologias existentes no mercado e ainda não utilizadas pelo conjunto de empresas de menor porte, dadas as especificidades destas últimas. A estrutura em redes de extensão do Sibratec é organizada regionalmente, buscando articular a infraestrutura de instituições de Ciência e Tecnologia (C\&T) existente para a prestação de serviços a partir da demanda das Micro, Pequenas e Médias Empresas (MPME) industriais e de serviços do país com recursos garantidos pelo Fundo Nacional de Desenvolvimento Científico e Tecnológico (FNDCT).

Este artigo busca então investigar, no estado do Rio de Janeiro, tanto a capacidade tecnológica das MPME industriais do setor metalmecânico, como o apoio oferecido pelo Sibratec no âmbito de uma de suas redes regionais de extensão tecnológica - a Sibratec-ET Rede RJ. O foco reside na demanda em TIB dessas empresas que se concentram na Zona Oeste (ZO) do Município do Rio de Janeiro (MRJ). Elas reúnem competências em caldeiraria, montagem industrial, corte-dobra e usinagem de metais, particularmente no que no que se refere a artefatos de aço inoxidável (MENDES, 2016). Esta localidade conta ainda com a presença de setores demandantes de produtos de aço inoxidável como alimentos e bebidas, químico, farmacêutico, editorial e gráfico, potencializando encadeamentos entre diversos setores produtivos (HASENCLEVER \& CUNHA, 2010). Vale dizer que o estudo contempla apenas as funções básicas de TIB, ou seja, normalização e regulamentação técnica, metrologia e avaliação de conformidade, esta última relacionada a aspectos de qualidade.

A literatura recente aponta a inovação como um elemento central do dinamismo econômico, mas estuda menos o aspecto da difusão tecnológica entre as MPME, perspectiva que ilumina a análise do programa de extensão tecnológica atual segundo arranjos regionais e de sua capacidade de apoio efetivo às MPME industriais em termos de modernização produtiva e organizacional, ou seja, de melhorias na sua capacidade tecnológica e na sua competitividade. Assim, além dessa introdução, o artigo está organizado em cinco seções. Na seção dois a metodologia adotada é apresentada e na seção três o referencial teórico sobre TIB em termos gerais e específicos. Na seção quatro são descritos os resultados empíricos obtidos e na seção cinco eles são analisados e discutidos, inclusive quanto a implicações de políticas. Na seção seis são apresentadas as considerações finais e a seguir as referências utilizadas.

\section{2- Metodologia}

Este artigo é o resultado de uma pesquisa de natureza qualitativa e empírica com finalidade exploratória baseada no método do estudo de caso. $O$ destaque da pesquisa qualitativa não é a quantidade de dados e descobertas, mas sua qualidade e profundidade a partir dos fenômenos estudados (TUZZO \& BRAGA, 2016). A pesquisa empírica difere da pesquisa pura por voltar-se menos para teorias e mais para sua aplicação, ainda que para obter uma visão geral e aproximativa dos fatos, dado seu caráter exploratório, enquanto o estudo de caso é voltado para um ou poucos objetos, de modo a permitir seu conhecimento amplo e ao mesmo tempo detalhado (GIL, 2011).

Yin (2010) acrescenta que o estudo de caso é o método de pesquisa adequado à compreensão de fenômenos contemporâneos e complexos em profundidade ao reunir múltiplas fontes de evidência e buscar seu encadeamento e convergência a partir do desenvolvimento anterior de proposições teóricas. Ele é indicado para investigar fenômenos individuais, grupais, organizacionais, sociais, econômicos e políticos. No campo da economia, o estudo de caso pode ser usado na análise de setores industriais, cidades ou regiões, de modo a permitir a apreensão das características holísticas e significativas de eventos da vida real como processos administrativos e organizacionais que acabam por refletir o grau de maturidade setorial, como se pretende mostrar aqui. 
No presente estudo foram utilizadas fontes bibliográficas, documentais e observacionais associadas a entrevistas pessoais. O cadastro da Federação das Indústrias do Estado do Rio de Janeiro (Firjan) foi a base para a seleção da amostra de empresas realizada a partir da Classificação Nacional de Atividades Econômicas (CNAE) do Instituto Brasileiro de Geografia e Estatística (IBGE), ambas referentes a 2014. Para contemplar o setor metalmecânico foram consideradas as empresas pertencentes à CNAE 2.0, Seção C (Indústrias de Transformação), Divisões 24 (Metalurgia), 25 (Fabricação de Produtos de Metal, exceto Máquinas e Equipamentos) e 28 (Fabricação de Máquinas e Equipamentos). Adicionalmente, foi considerado o número de ocupações que resultou na seleção de 59 MPME localizadas na ZO do MRJ, isto é, com até 499 empregados. Contudo, gestores e especialistas de apenas 23 empresas concordaram em participar das entrevistas realizadas com o auxílio de um questionário semiestruturado.

Foram realizadas ainda quatro entrevistas pessoais em profundidade por pautas ou temas com gestores e especialistas da Sibratec-ET Rede RJ por meio de um roteiro, de modo a captar suas opiniões e percepções (GIL, 2011). A partir das demandas tecnológicas e práticas organizacionais da indústria identificadas, bem como das informações sobre o funcionamento da Sibratec-ET Rede RJ obtidas, pretendeu-se verificar o apoio efetivo desta última à capacitação em TIB das MPME da região. Tratava-se de responder à seguinte questão de pesquisa: a Sibratec-ET Rede RJ é adequada para apoiar o desenvolvimento produtivo e inovativo das MPME da ZO do MRJ em relação à TIB? Como destaca Yin (2010), as evidências observacionais e as entrevistas constituem as principais fontes dos estudos de caso.

\section{3- Referencial Teórico}

Como o desenvolvimento de proposições teóricas é necessário para orientar a coleta e análise de dados empíricos, esta seção apresenta o referencial teórico sobre TIB, dividindo-se em duas partes. Na primeira são abordados os aspectos gerais destas tecnologias infraestruturais que são seguidos pela literatura que trata mais especificamente das funções de normalização e regulamentação técnica, de metrologia e de avaliação de conformidade que envolve aspectos de qualidade. Cabe destacar que a infraestrutura de TIB apresenta especificidades em países em desenvolvimento como o Brasil, conforme é assinalado a seguir.

\section{1- Aspectos Gerais de TIB}

Um conjunto de tecnologias - que está na base do processo industrial e que é considerado infraestrutural para as empresas - permeia os processos desde a etapa do projeto - que envolve conceito, planejamento, engenharia e produção piloto - até a introdução no mercado e efetiva produção de produtos, com padrões de desempenho e características que atendem às demandas dos compradores. Tal conjunto de atividades técnicas pressupõe a implantação de um sistema baseado no estudo científico de aspectos tecnológicos interrelacionados que têm importância econômica significativa (GALLINA, 2009; MADEIRA, 2009; SHAPIRA, 1990; TIGRE, 2014).

Em que pese o enfoque restrito que relaciona TIB apenas a aspectos de produtos, seu escopo é estratégico por envolver vantagens competitivas, tanto no âmbito macroeconômico, como microeconômico (FLEURY, 2007). Como destaca o autor, a TIB tem fronteiras amplas, com sua gênese na ciência básica voltada à criação de conhecimentos para sustentar o avanço tecnológico. Esta concepção ampla do autor, seus fatores de influência e seu papel na economia globalizada encontram-se representados na Figura 1. 
Figura 1:TIB e Desenvolvimento Industrial em Países Desenvolvidos eem Países em Desenvolvimento

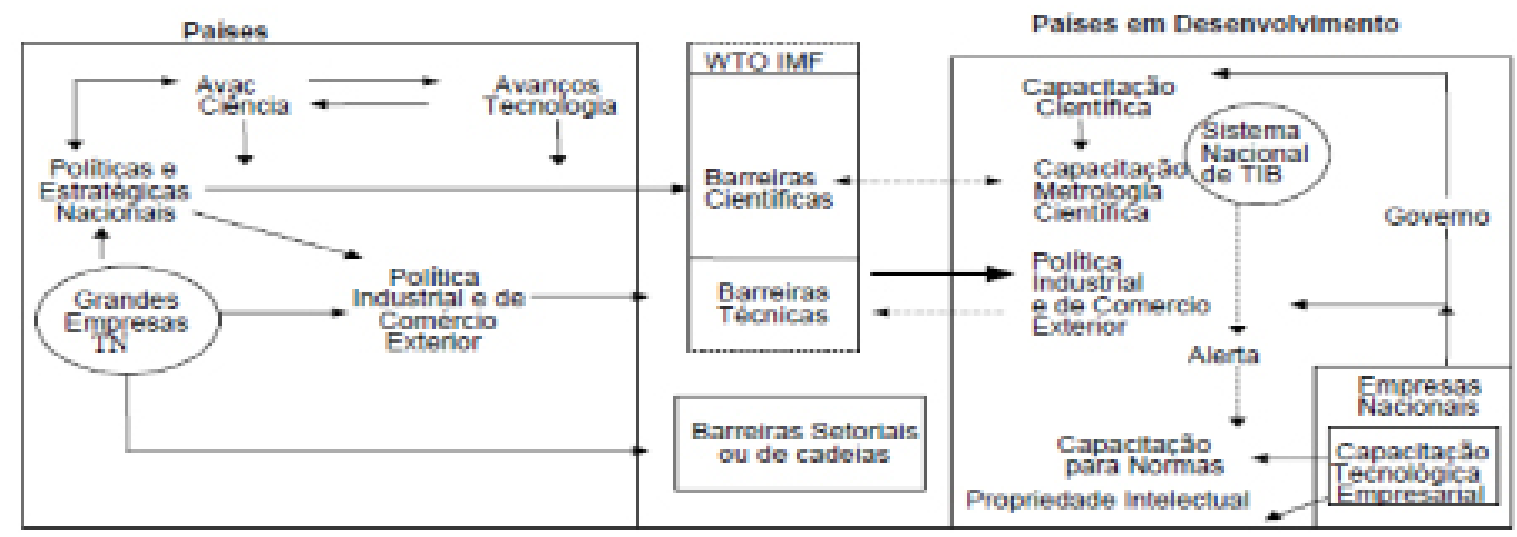

Fonte: Fleury (2007).

Outrossim, a TIB guarda relação com as políticas de Ciência e Tecnologia (C\&T) e de educação dos países, com repercussões na política de comércio exterior, o que tem se verificado especialmente nos dias de hoje. Embora o termo TIB tenha sido concebido no Brasil no final dos anos 70, a estruturação de suas atividades ocorreu no âmbito do Programa de Apoio ao Desenvolvimento Científico e Tecnológico (PADCT) criado em 1984 e lançado em 1985 (PADCT-TIB), possibilitando importantes avanços em TIB a nível nacional (SOUZA, 2000).

Em 2001 a concepção de TIB se ampliou ao incorporar dois subprogramas: Infraestrutura de TIB e ServiçosTecnológicos para InovaçãoeCompetitividade.Oprimeiroenvolviaasáreas denormalização e regulamentação técnica, metrologia e avaliação de conformidade, além de tecnologias de gestão, propriedade intelectual e informações tecnológicas (BRASIL, 2001). O segundo era voltado para Instituições de Pesquisa e Desenvolvimento Tecnológico, Reorientação Estratégica e Modernização Gerencial, Consolidação e Ampliação de Linhas de Atuação, Entidades Tecnológicas Setoriais (ETS), Viabilidade Técnica e Econômica para Novas ETS e Consolidação de ETS ${ }^{3}$. A ideia era vincular TIB à inovação, ou seja, fortalecer as atividades de Pesquisa, Desenvolvimento, Engenharia e Serviços Tecnológicos em apoio ao desenvolvimento produtivo e inovativo industrial.

Os aspectos históricos destas atividades e processos no país foram descritos por Souza (2000), Abreu (2005) e Dias (2007). Trata-se de uma infraestrutura tecnológica de apoio ao desenvolvimento industrial com a necessária ressonância que cada um dos aspectos tecnológicos abrangidos pela TIB deve ter nas empresas industriais. Como assinala Oliveira (2015), tais aspectos precisam ser incorporados, tanto na formação profissional, sobretudo de engenheiros, como nas rotinas de trabalho de empresas de diferentes portes e setores econômicos, em especial as MPME. A autora distingue as funções essenciais de TIB como normalização, regulamentação técnica, metrologia e avaliação de conformidade das funções conexas de prestação de serviços de infraestrutura tecnológica que incluem tecnologias de gestão, propriedade intelectual e informações tecnológicas. ${ }^{4}$ (BRASIL, 2001).

As tecnologias de gestão tiveram sua importância substantivada no Brasil no período recente com a constituição da base institucional em gestão da qualidade culminando com a criação em 1987 do Projeto de Especialização em Gestão da Qualidade (PEGQ) no âmbito do Subprograma TIB do PADCT. Do ponto de vista conceitual, essas tecnologias abrangem um conjunto de metodologias

3 O presente artigo analisa apenas a demanda por TIB na sua concepção original que na pesquisa envolveu o enfoque do estado de maturidade ou de implantação de sistemas de gestão da qualidade e aspectos de certificação de sistemas de gestão fabril. Eles permitem avaliar a demanda das empresas por metrologia, normalização, regulamentação técnica e avaliação da conformidade. Outros aspectos da TIB podem ser encontrados no estudo de Mendes (2016). 
e técnicas de gerenciamento sistematizado para o desdobramento dos objetivos estratégicos e operacionais das organizações nos ambientes em que atuam (BRASIL, 2001). Trata-se de processos organizacionais estruturados e rotinizados voltados para modernização gerencial, melhoria de qualidade, aumento de competitividade e sustentabilidade. Eles abrangem sistemas de gestão ambiental, de qualidade, de segurança de alimentos e de responsabilidade social que contam com normas técnicas nacionais e internacionais (BRASIL, 2001; OLIVEIRA \& SOUZA, 2014).

A propriedade intelectual engloba um conjunto de criações imateriais, estando relacionada normativamente à garantia de direitos de uso das criações pelos autores. Ela confere aos titulares do direito a liberdade de impedir o uso da criação por terceiros. Durante o prazo legal estipulado, o titular do direito de propriedade intelectual pode explorar economicamente bens e processos produtivos decorrentes deste direito, de acordo com a delimitação definida em lei. O sistema de propriedade intelectual envolve: (i). Direitos de Autor (DA) e Conexos (DC) - obras literárias, artísticas e científicas (DA), interpretações artísticas e execuções, fonogramas e transmissões por radiodifusão (DC) e ainda programas de computador; (ii).Propriedade Industrial - patentes, desenho industrial, marcas, indicação geográfica; e (iii).Direitos Sui Generis - proteção de novas variedades de plantas, topografia de circuito integrado, conhecimentos tradicionais e manifestações folclóricas (OMPI-INPI, 2018).

As informações tecnológicas normalmente contidas em documentos de patentes permitem investigar o que já foi desenvolvido em determinada área, as rotas tecnológicas usadas e outros dados importantes para quem precisa inovar. Para a inserção competitiva no mercado, as empresas também podem utilizar o sistema patentário como fonte de informações tecnológicas, uma vez que os documentos de patente contêm dados que permitem identificar o estado da técnica de uma determinada tecnologia desvendando tendências tecnológicas, monitorar investimentos em Pesquisa e Desenvolvimento (P\&D) de empresas concorrentes, recursos humanos com determinadas competências, além de outras informações de interesse estratégico (CRESPO \& SOUZA, 2006).

Portanto, as informações tecnológicas representam conhecimentos científicos, empíricos ou intuitivos, relacionados ao modo de produzir um produto ou de prestação de um serviço oferecido ao mercado, envolvendo ainda informações para a indústria e sobre a indústria. Neste rol de conhecimentos, podem ser relacionadas as tecnologias de processos, produtos e gestão. É também o elemento-chave no processo de mudança e inovação do setor industrial, visando à competitividade (BRASIL, 2001). A boa gestão de informações tecnológicas no âmbito de empresas industriais permite a exploração de vantagens competitivas, dada a possibilidade de previsão de ameaças e oportunidades de mercado, contribuindo para a redução das incertezas do ambiente externo (OLIVEIRA \& SOUZA, 2014).

Tironi (2015) mostra a correlação positiva entre TIB e inovação, não apenas porque a TIB é fonte de informações tecnológicas básicas, mas sobretudo por ser um elemento que favorece a cooperação para a inovação ${ }^{5}$. A capacitação em TIB faz parte do que Penrose (2006) define como coleção de recursos produtivos das empresas. Como a maior parte dos esforços de aprendizagem tecnológica e de inovação é feita por meio da combinação de fontes internas e externas, a capacidade de absorção torna-se necessária (COHEN \& LEVINTHAL, 1989, 1990). As empresas precisam ainda construir e acumular capacitações tecnológicas (BELL \& PAVITT, 1993) e identificar suas capacitações dinâmicas, ou seja, aquelas que alavancam inovações (TEECE, 2007; TEECE et al., 1997).

Assim, a internalização da infraestrutura de TIB por parte das empresas fornece a base para os processos de melhoria contínua necessários à elevação do seu patamar de competitividade e de capacidade inovativa. A TIB compreende ativos físicos, técnicas, procedimentos e metodologias

$5 \quad$ Uma visão mais ampla das fontes de informação tecnológica para as empresas do setor metalmecânico da ZO do MRJ pode ser obtida a partir da leitura do estudo de Mendes (2016). 
de codificação, análise e normalização de produtos e processos e é este conjunto de capacidades tecnológicas ou competências fundamentais que dá suporte ao progresso técnico. Destacam-se entre as funções básicas de TIB a normalização, a regulamentação técnica (autorização, aprovação, registro, licença e homologação), a metrologia e a avaliação de conformidade (acreditação, inspeção, ensaios, certificação e suas funções correlatas) segundo padrões de qualidade. Tais competências ou especificidades de TIB são suscintamente apresentadas a seguir.

\section{2- Aspectos Específicos de TIB}

A normalização se relaciona à necessidade de padronização decorrente da crescente ampliação do mercado mundial para novos produtos e serviços. A norma técnica pode ser considerada a tradução, em termos de requisitos técnicos, de um consenso social em torno de expectativas sobre o desempenho de produtos, serviços e sistemas, estando em geral associada à expectativa de qualidade de tais elementos (ABREU, 2005). O sistema normativo é estruturado hierarquicamente, indo desde a base com a normalização empresarial seguida da nacional, chegando ao nível regional e alcançando, no topo deste encadeamento hierárquico, a normalização internacional que representa interesses comuns entre países (ABNT, 2018).

A literatura discute os ganhos advindos da harmonização de especificações de produtos e serviços provenientes da redução de incertezas nas relações industriais e comerciais, bem como de desperdícios, facilitando o intercâmbio com os consumidores nacionais e internacionais (DIAS, 2007; OLIVEIRA, 2011). Tal arcabouço normativo e regulatório caracteriza um ambiente minimamente adequado às atividades de estruturação de processos produtivos e de comercialização de produtos.

Como destaca Tironi (2017), a normalização favorece a inovação ao facilitar o acesso ao conhecimento tecnológico e estimular a escala econômica por meio da ampliação de mercados, da geração de estímulo incremental às inovações e do crescimento do interesse por regulamentações e normas técnicas. A fragmentação e dispersão das cadeias produtivas pelo mundo e a globalização dos mercados são fenômenos com os quais as empresas lidam no cenário competitivo em que buscam atuar e que exigem preparo em diversos níveis, ou seja, capacidade tecnológica.

A regulamentação técnica é um documento aprovado por órgãos governamentais contendo características de produtos, processos e métodos de produção, bem como disposições administrativas obrigatórias que podem incluir prescrições quanto a terminologias, símbolos, embalagens, marcações ou etiquetagens ou delas tratar exclusivamente. Diferentemente da regulamentação técnica, cuja adoção é obrigatória, a norma técnica advém de concertação em âmbito setorial, tendo em vista o consenso em torno da adoção de um dado padrão ou de certo tipo de uso disseminado socialmente, tendo caráter voluntário. Trata-se de um documento aprovado por instituição reconhecida que prevê regras, diretrizes ou características para produtos, processos e métodos de produção e que também pode incluir prescrições ou delas tratar exclusivamente (INMETRO, 2018).

A metrologia, por sua vez, é a ciência que abrange aspectos teóricos e práticos relativos a medições, dada a incerteza existente em qualquer campo da ciência ou da tecnologia (GONZÁLEZ \& VÁZQUEZ, 1996). No Brasil, a Lei n 5.966/1973 criou o Sistema Nacional de Metrologia, Normalização e Qualidade Industrial (Sinmetro), o Conselho Nacional de Metrologia, Normalização e Qualidade Industrial (Conmetro) e o Instituto Nacional de Metrologia, Qualidade e Tecnologia (Inmetro), cabendo a este último promover junto à sociedade a confiança em medições e produtos por meio de atividades de metrologia e avaliação de conformidade, buscando ainda a harmonização entre as relações de consumo, a inovação e a competitividade das empresas que atuam no país (INMETRO, 2018). 
Nessa estrutura sistêmica e articulada, o Inmetro atua com o objetivo de fortalecer as empresas de base nacional, isto é, ele atua como instrumento de melhoria da competitividade nacional. $O$ Brasil adota a Metrologia Científica e Industrial voltada para a expansão das atividades produtivas e das inovações tecnológicas, o que necessariamente envolve a criação de uma infraestrutura favorável ao fortalecimento das empresas de base nacional. Trata-se de fazer frente às atuais barreiras técnicas à exportação que surgem em decorrência da falta de harmonia dos sistemas técnicos dos países no âmbito de suas transações comerciais. Portanto, seu escopo de atuação é estratégico, conforme assinalado por Fleury (2007).

Assim, a metrologia tem impactos diretos sobre os processos produtivos. A prática das empresas de controle metrológico deve exceder a calibração e a manutenção de instrumental de medição referindo-se, tanto ao uso de um conjunto de ações de garantia de conformidade de seus produtos, como a tolerâncias de vários tipos: geométricas, dimensionais, de cor, volume, dureza, resistência etc. Estas ações não geram alteração de características, mas têm papel fundamental na garantia da qualidade de produtos de qualquer empresa industrial, constituindo elementos básicos para a implantação de sistemas de qualidade (OLIVEIRA, 2008).

Por outro lado, o grau de conformidade com parâmetros especificados é um critério de comprovação da qualidade estabelecido entre fornecedores e clientes ao acordarem previamente o intercâmbio de produtos que devem satisfazer suas especificações de projeto (OLIVEIRA, 2011). Algumas definições clássicas de qualidade giram em torno da conformidade ou adequação ao uso, embora a que se firmou por se mostrar mais adequada ao ambiente de negócios contemporâneo é a que relaciona qualidade a percepções positivas que refletem a satisfação dos clientes.

A qualidade se materializa por meio de especificações técnicas em geral encontradas em boletins e desenhos técnicos, enquanto a conformidade é identificada por comparação de características desejadas com padrões ou por sua aferição mediante o uso de instrumentos e equipamentos específicos. As diretrizes do Sistema Brasileiro de Avaliação da Conformidade (SBAC) indicam o reconhecimento do atendimento aos requisitos especificados em termos nacionais (OLIVEIRA, 2011). Vale acrescentar no âmbito do Sinmetro a criação da Rede Brasileira de Laboratórios de Ensaios (RBLE) visando o provimento de serviços de avaliação de conformidade por meio de ensaios.

A certificação está relacionada à avaliação de conformidade de produtos e processos para conferir credibilidade quanto à adequação de sistemas de produção a requisitos de segurança e padronização previamente definidos, sendo realizada por uma terceira parte independente. Vale destacar que as empresas hoje buscam ir além da conformidade e da criação de valor para os acionistas para atender às necessidades de todos os stakeholders como empregados, fornecedores e clientes, além da comunidade em geral e do ambiente natural. Trata-se da sustentabilidade corporativa como evolução do movimento da responsabilidade empresarial em cumprimento aos padrões de desempenho sustentável (BENN, 2007; BHIMANI \& SOONAWALLA, 2005).

As organizações certificadoras são acreditadas por organismos internacionais comoa International Organization for Standardization (ISO) e a Occupational Health and Safety Assessment Series (OHSAS) e nacionais como a Associação Brasileira de Normas Técnicas (ABNT). Esta última é responsável pela elaboração das normas brasileiras (ABNT NBR) e atua, desde 1950, na avaliação de conformidade e certificação de produtos, sistemas e rotulagem ambiental, contribuindo para a implementação de políticas públicas, o desenvolvimento de mercados, a segurança dos cidadãos e a defesa dos consumidores brasileiros (ABNT, 2018).

Considera-se, pois, a necessidade de uma gestão sistêmica de TIB no âmbito das empresas, dado seu caráter infraestrutural e as imbricações entre normalização, metrologia e avaliação de conformidade. A TIB é crítica para as empresas ao melhorar suas possibilidades de aprendizagem tecnológica, de construção de capacidades tecnológicas e de geração de inovações. O pleno 
domínio dessas tecnologias infraestruturais não é uma opção, mas um requisito básico para a sobrevivência das empresas industriais, não apenas no mercado doméstico, mas sobretudo internacional, dadas as barreiras técnicas impostas atualmente.

\section{4- Resultados da Pesquisa}

Esta seção apresenta os resultados da pesquisa de campo realizada, sendo distribuída em três partes. A primeira mostra a demanda de TIB no setor metalmecânico segundo as opiniões e percepções dos gestores e especialistas entrevistados. A segunda aborda os aspectos de implementação da Sibratec-ET Rede RJ também de acordo com os gestores e especialistas entrevistados aos quais se agregaram, em caráter complementar, fontes bibliográficas e documentais. A correlação entre a demanda do setor e a oferta de apoio é então estabelecida na terceira parte.

\section{1- O Lado da Demanda: Capacitação em TIB no Setor Metalmecânico}

O que os resultados de modo geral indicaram foi a fragilidade de capacitação em termos de TIB da maioria das empresas. De fato, à medida que as questões sobre a implantação de sistemas de gestão foram sendo formuladas, desde os mais simples aos mais complexos que requerem maior grau de capacitação em TIB, reduziu-se o número de MPME que informaram possuir tais sistemas. Verificou-se ainda que a certificação de sistemas organizacionais é um ponto fraco para grande parte das empresas entrevistadas, como mostra a Tabela 1.

Tabela 1: Distribuição de Capacitações em TIB

\begin{tabular}{|c|c|c|}
\hline Tipo de Capacitação & Empresas (no) & Empresas (\%) \\
\hline Inspeção Final de Produtos Implantada & 22 & 95,7 \\
\hline Controles de Processos Implantado & 13 & 56,5 \\
\hline Sistemas de Gestão da Qualidade Implantados & 11 & 47,8 \\
\hline Sistemas de Gestão da Qualidade Certificados & 8 & 34,8 \\
\hline Sistemas de Gestão Ambiental Certificados & 3 & 13,0 \\
\hline Sistemas de Gestão de Saúde e Segurança Certificados & 2 & 8,7 \\
\hline Certificações Específicas: Petrobras, Shell, PDVSA e outras. & 1 & 4,3 \\
\hline
\end{tabular}

Fonte: Adaptação de Mendes (2016).

Para garantir um nível mínimo de inspeção em relação aos parâmetros de produção especificados de acordo com as exigências dos mercados atendidos, 95,7\% das empresas informaram possuir sistemas de inspeção final de produtos, ou seja, apenas uma empresa informou não possuir tal sistema, embora tenha declarado possuir sistema de garantia da qualidade, o que contempla a inspeção.

Por outro lado, $56,5 \%$ das empresas afirmaram possuir controle de qualidade de processos produtivos implantados que melhoram a confiabilidade e o desempenho dos mesmos, evitando o foco na detecção de erros e na retificação característico da inspeção ao final do processo produtivo que enfatiza a verificação de produtos. Contudo, apenas $47,8 \%$ das empresas declararam possuir sistemas de gestão da qualidade implantados que permitem garantia de qualidade ou que podem ser caracterizados, mais propriamente, como sistemas de qualidade envolvendo medição de custos de qualidade, solução de problemas e planejamento de qualidade.

Quando questionadas sobre sistemas de gestão de qualidade certificados, um número ainda menor de empresas respondeu afirmativamente, ou seja, 34,8\% das MPME entrevistadas declarou possuir sistemas de gestão da qualidade certificados, o que lhes permitiria maior 
credibilidade junto a seus mercados, uma vez que este tipo de certificação tende a se tornar uma exigência mínima de fornecimento em cadeias mais amplas, isto é, que incluem outros setores, tanto no país, como no exterior.

Quanto aos sistemas de gestão ambiental certificados, apenas $13 \%$ das empresas responderam afirmativamente, enquanto $8,7 \%$ declararam possuir sistemas de saúde e segurança do trabalho certificados, o que representou um total de apenas duas empresas. Vale mencionar que quando estes dados foram analisados levando em conta o porte das empresas, observou-se uma fragilidade maior de capacitação em TIB nas empresas de menor porte, isto é, com até 19 empregados. Contrariamente, as empresas de médio porte foram as que apresentaram os melhores resultados em termos de capacitação em TIB.

\section{2- O Lado da Oferta: a Sibratec-ET Rede RJ}

A extensão tecnológica no Brasil foi criada com base na experiência exitosa da extensão agrícola. As primeiras iniciativas de política industrial voltadas para a extensão tecnológica se deram com o lançamento do PADCT-TIB em 1985 e do Programa da Qualidade e Produtividade (ProQP) em 1986 (DARÓS, 1997; DIAS, 2007). Depois deles, inexistiram no país sistemas estruturados para estes fins. Ainda assim, surgiram alguns programas isolados buscando capacitar as empresas, principalmente as micro e pequenas ${ }^{6}$ em aspectos específicos de TIB. Os principais programas nacionais com características de extensão industrial de caráter continuado foram o Projeto Extensão Industrial Exportadora (Peiex), o Programa Sebrae de Consultoria Tecnológica (Sebraetec), o Programa de Unidades Móveis (Prumo), o Programa de Apoio à Exportação (Progex) e o Programa Inovar para Competir.

Contudo, Madeira (2009) revela que os resultados da aplicação desses programas de extensão em apoio às demandas das MPME não vinham sendo satisfatórios. A descontinuidade das ações foi identificada como uma de suas principais deficiências, bem como a debilidade ou ausência de mecanismos institucionais de coordenação visando uma definição melhor de diretrizes, ao lado de uma possível articulação de ações entre os programas de extensão. Com base no trabalho de Shapira (1990), a autora mostra que tais falhas não estiveram presentes nos modelos de apoio japoneses e norte-americanos, os quais dispunham de processos de atuação continuada. No Brasil houve comprometimento com a geração de resultados pela restrição do escopo dos serviços realizados, com impacto direto na evolução da base de conhecimento dos produtores locais.

Porém, a criação do Sibratec em 2007 representou uma mudança na política de extensão industrial no país. Composto por um comitê gestor, três comitês técnicos e uma secretaria executiva ligada ao atual Ministério da Ciência, Tecnologia, Inovações e Comunicações (MCTIC), ele é operado nacionalmente pela Financiadora de Estudos e Projetos (Finep) e tem como prioridade a promoção de inovações tecnológicas nas empresas. Tratava-se de intensificar o apoio tecnológico às empresas, bem como as ações de fomento à inovação. A Figura 2 apresenta a estrutura concebida para o Sibratec.

$6 \quad$ Alguns programas envolviam o atendimento também a empresas de médio porte. 


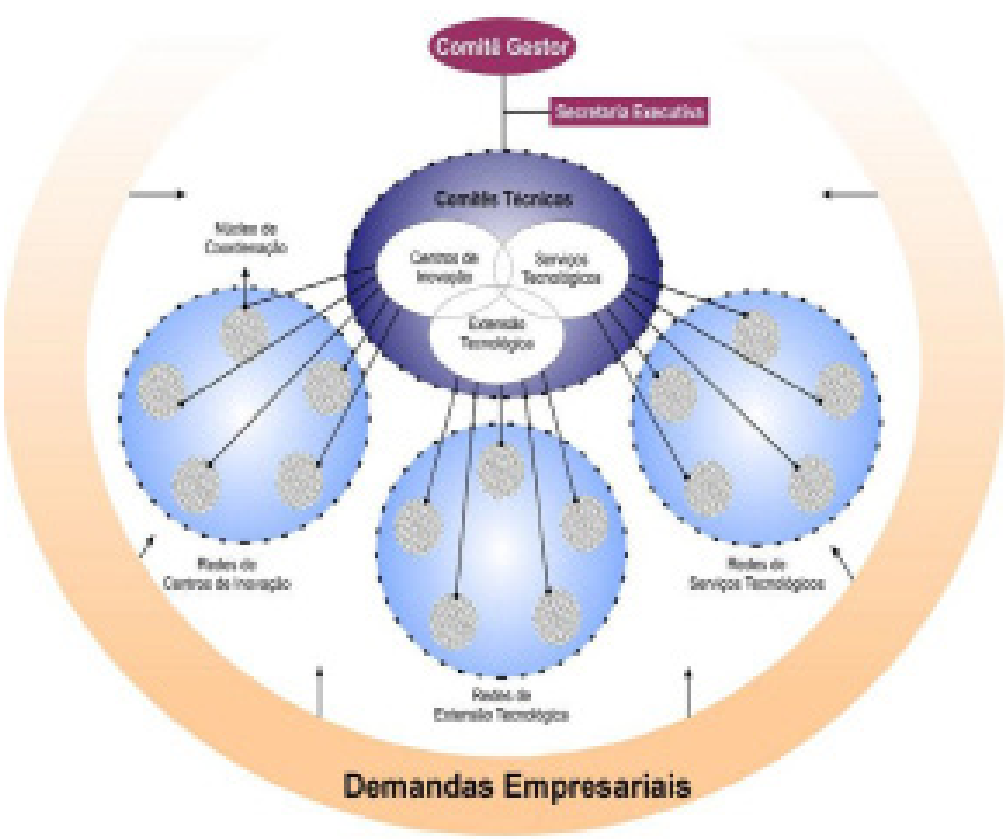

Fonte: Sibratec (2018).

O Sibratec é um programa estruturado por meio de três redes articuladas dentre as quais a rede de extensão tecnológica que possui amplo escopo de atuação, buscando superar as inconsistências de iniciativas isoladas anteriores, bem como melhorar a eficiência e eficácia no uso dos instrumentos projetados (MADEIRA, 2009; MENDES \& HASENCLEVER, 2015).

Estruturada em bases regionais, a Sibratec-ET Rede RJ foi criada em 2009 e entrou em operação em 2010 a partir de organizações com funções previamente definidas e consideradas prioritárias como o Instituto Nacional de Tecnologia (INT) encarregado de ser o coordenador técnico do projeto e a Rede de Tecnologia e Inovação do Rio de Janeiro (Redetec) responsável pela articulação com as Instituições Científicas, Tecnológicas e de Inovação (ICT) e pela gestão administrativa e financeira desse arranjo institucional.

O Serviço Brasileiro de Apoio às Micro e Pequenas Empresas (Sebrae) foi responsável pelo apoio às MPME e pelo aporte de recursos financeiros correspondente a $10 \%$, enquanto a Fundação de Amparo à Pesquisa do Estado do Rio de Janeiro (Faperj) na condição de órgão estadual de fomento foi também responsável pelo aporte de $10 \%$ de recursos financeiros. A Financiadora de Estudos e Projetos (Finep) como operadora nacional e gestora financeira estratégica do Programa forneceu a maior parte dos recursos oriundos do FNDCT, ou seja, $70 \%$. Os $10 \%$ restantes foram considerados como contrapartida das empresas atendidas pelo Programa. Com relação ao arranjo Sibratec-ET Rede RJ, uma síntese dos relatos dos entrevistados acerca de sua implementação é resumida no Quadro 1. 


\section{Caracterização}

1. O Programa foi "puxado" pela demanda em termos de capacidade de atendimentos com dimensionamento inicial de cerca de 10 atendimentos-empresa por mês.

2. Foram utilizadas cinco modalidades de atendimento.

3. As modalidades tinham especificidades próprias, mas com o instrumento relativamente aberto, ajustável à demanda das empresas demandantes dos serviços de apoio.

4. A eficácia foi baixa, uma vez que a execução dos atendimentos sofreu muitas intermitências ao longo do período de vigência do arranjo institucional Sibratec-ET no estado do Rio de Janeiro.

5. Houve necessidade de repactuação do convênio ao longo da execução do Programa Sibratec-ET com ajustes inclusive das metas pactuadas inicialmente;

6. Não houve atendimento na ZO do MRJ (recorte que inclui apenas Bangu, Realengo, Campo Grande e Santa Cruz), território da pesquisa da demanda por capacitação tecnológica das MPME industriais do setor metalmecânico.

7. Não houve estratégia específica de divulgação do Programa Sibratec-ET. Esta foi constituída de forma não pactuada, informal, com apoio em dado momento da estrutura do INT e apenas com a criação de folder de divulgação.

8. O programa não atingiu as metas originais no período previsto inicialmente no convênio, apresentando número de atendimentos muito baixo frente ao tempo de execução do Programa no ERJ gerando como consequência a baixa eficiência.

9. Houve conflitos na operação dos instrumentos de extensão, ou seja, entre a Sibratec-ET e Sebraetec, dada a similaridade e concorrência potencial de ações no estado, sendo necessário um acordo na governança da Sibratec-ET para que as atividades e operações de ambos se tornassem complementares visando otimizar o uso de recursos e gerar menos desgastes e sobreposições de ações, o que foi obtido, em parte, durante certo tempo, apesar da perda de vigor da Sibratec-ET.

Fonte: Adaptação de Mendes (2016).

Como fica claro na síntese apresentada, o resultado da implantação da Sibratec-ET Rede RJ não foi bom, em que pese o fato de sua concepção ter representado um avanço em relação aos programas de extensão tecnológica anteriores. Esta conclusão foi corroborada pelo Relatório Técnico-Gerencial Final elaborado pelo INT na condição de coordenador técnico da proposta (INT, 2016). Assim, fica evidenciada a falta de efetividade deste arranjo institucional de apoio ao desenvolvimento produtivo das MPME na ZO do MRJ. Conforme apontado por Mendes (2016), as empresas da região não receberam qualquer visita extensionista no período de 2009 e 2016 de vigência da Sibratec-ET Rede RJ.

\section{3- Demanda versus Oferta: Capacitação em TIB versus Apoio Institucional}

Os resultados da pesquisa no setor metalmecânico da ZO no MRJ revelaram a baixa capacitação em TIB das empresas da região, tendo em vista que a grande maioria delas possui apenas sistemas de inspeção final de produtos implantados e, em menor escala, controles de processos produtivos, além de apresentarem baixo índice de certificação, o que lhes poderia trazer benefícios de imagem e de posicionamento competitivo, além de melhores possibilidades de inserção em cadeias produtivas envolvendo outros setores.

O Quadro 2 permite a análise da situação das empresas pesquisadas que reflete sua demanda por capacitação em TIB em relação à oferta de apoio institucional por intermédio da rede de extensão encarregada de atuar no estado do Rio de Janeiro - a Sibratec-ET Rede RJ. Verifica-se a existência de uma correlação positiva em termos de implementação, apesar da baixa eficiência e eficácia dos resultados obtidos que acabou por significar baixa efetividade. 
Quadro 2: Demanda versus Oferta: Capacitação em TIB versus Apoio Institucional

\begin{tabular}{|c|c|c|c|c|}
\hline Item & Elementos & MPME (\%) & Demandas Gerais & Oferta Sibratec-ET \\
\hline 1 & \multirow{4}{*}{ TIB } & $50-70$ & Implantação de Sistemas de Gestão da Qualidade & Sim \\
\hline 2 & & $20-40$ & Certificação de Sistemas de Gestão Industrial & Sim \\
\hline 3 & & 20-30 & $\begin{array}{l}\text { Implantação de Laboratórios para Análise de Confor- } \\
\text { midade de Produtos }\end{array}$ & Sim \\
\hline 4 & & $10-20$ & $\begin{array}{c}\text { Acesso a Laboratórios Externos para Inspeção } \\
\text { e Ensaios }\end{array}$ & Sim \\
\hline
\end{tabular}

Fonte: Adaptação de Mendes (2016).

Ainda que não tenha havido qualquer atendimento ao conjunto de empresas pesquisadas, as possibilidades do Sibratec via rede de extensão tecnológica contempla as demandas apresentadas pelas empresas, com possibilidade de oferta também pela rede de serviços tecnológicos do Sibratec que atua em sintonia com as redes estaduais de extensão, dado que há interação dos comitês gestores no âmbito deste programa a nível nacional (BRASIL, 2007).

\section{5- Análise, Discussão e Implicações}

Esta seção apresenta os comentários sobre as evidências obtidas a partir das várias fontes utilizadas, dividindo-se em duas partes. A primeira oferece a análise e discussão dos resultados da pesquisa de campo, tanto do ponto de vista da capacidade atual em TIB das empresas investigadas e sua demanda futura, como do ponto de vista da oferta de apoio no âmbito da Sibratec-ET Rede RJ. Na segunda parte são levantadas algumas implicações de políticas decorrentes desse contexto.

\section{1- Análise e Discussão de Resultados}

Do lado da demanda, verificou-se uma fragilidade generalizada quanto à capacitação em TIB nas MPME pesquisadas, ou seja, a situação é de debilidade e até mesmo de falta de competências mínimas, pois apenas 50\% delas têm controle de processos implantados formalmente. Este é um requisito mínimo de capabilidade de processos, isto é, de métricas que garantem a qualidade industrial visando a entrega de valor aos futuros clientes. A pesquisa evidenciou a garantia de inspeção de produtos em quase todas as empresas entrevistadas, o que demonstra competência operacional de produção, ainda que sem o respaldo da capabilidade de processos, comprometendo o desempenho em termos de qualidade e confiabilidade.

As empresas do setor metalmecânico pesquisadas têm o domínio técnico de fabricação de produtos, dada sua habilidade histórica em fazê-los por meio de técnicos qualificados, o que sugere que os conhecimentos encontram-se mais no nível tácito intrínseco aos indivíduos, não sendo apropriados pelas mesmas em suas rotinas, seja do ponto de vista das especificações técnicas dos produtos, seja do ponto de vista dos processos fabris propriamente ditos, não existindo, portanto, o domínio de rotinas de gestão do conhecimento tecnológico. Verificouse ainda a participação decrescente das empresas em relação à certificação de seus sistemas de gestão, exceção feita apenas às empresas de médio porte.

Outro aspecto a ser destacado diz respeito à percepção dos gestores sobre a necessidade de conhecimentos relacionados à TIB: em geral ela foi menor, chegando a ser mínimo entre as microempresas, ou seja, evoluindo de maneira crescente e proporcional ao porte das empresas, o que pode estar relacionado ao nível de exigência dos clientes e mercados nos quais elas atuam que é mais alto, significando garantias mais explícitas e rigorosas de qualidade. As maiores exigências foram apontadas nos mercados em que sistemas de gestão 
de processos industriais certificados é um requisito obrigatório nas transações comerciais ${ }^{7}$.

Do lado da oferta, a pesquisa mostrou que a Sibratec-ET Rede RJ possui modelagens adequadas de atendimento à demandas das MPME do setor metalmecânico localizadas na ZO do MRJ, o que permite concluir que há uma aderência entre a oferta de apoio institucional na vertente da extensão tecnológica e a capacitação em TIB das empresas. Porém, o arranjo institucional não foi efetivo quanto a seus fins, uma vez que o apoio ao desenvolvimento das empresas não ocorreu na proporção necessária. Contudo, a demanda identificada revela oportunidades de apoio institucional para construção da capacitação em TIB, já que as empresas, em sua maioria, não tem esta capacidade tecnológica incorporada em suas rotinas.

O atual modelo de apoio é recente e deve ser aprimorado, sobretudo no que tange à coordenação das atividades. A rede de extensão tecnológica estadual tampouco apresentou fluxos de recursos financeiros e de informações entre os parceiros capazes de sustentar as ações de atendimento na quantidade e qualidade adequadas durante o longo período de 2009 a 2016. Os resultados finais foram atingidos por repactuações e pela manutenção de uma meta de atendimentos muito aquém do potencial e da demanda do estado do Rio de Janeiro, problema identificado em 2013 também em outras redes de extensão em implementação no país.

No caso do Rio de Janeiro, este problema não foi superado até o fim da operação da Sibratec-ET Rede RJ em fevereiro de 2016 (INT, 2016; BRASIL, 2013; MENDES, 2016). Embora a implementação da Sibratec-ET Rede RJ não tenha sido bem sucedida, existem outros estados que obtiveram melhores resultados como Minas Gerais, Paraná e Santa Catarina que poderiam ser analisados para divulgação e referência para aplicações futuras.

\section{2- Implicações de Políticas}

A necessidade de capacitação em TIB das empresas do setor metalmecânico localizadas na ZO do MRJ ratifica a necessidade de apoio institucional via extensão tecnológica. Tal apoio deve ocorrer por sistema externo, haja vista a falta de capacidade endógena das empresas para tal mister. Cabe, portanto, uma pergunta: as empresas sabem que precisam desenvolver competências em TIB? A resposta vai no âmago da questão da difusão do conhecimento tecnológico básico existente no país (e do seu impacto nos processos fabris), mas que não flui de forma adequada não atingindo, por exemplo, as MPME aqui analisadas. A divulgação da infraestrutura de serviços tecnológicos existente no país e no estado do Rio de Janeiro é fundamental para as MPME pesquisadas que demonstraram desconhecimento até mesmo de suas necessidades básicas.

Como o segmento das MPME é heterogêneo, o apoio tecnológico deve considerar distintas abordagens de atendimento às empresas locais: um grupo é constituído por empresas de menor porte que ainda realizam muitos processos manualmente; outro grupo é formado pelas MPME que, que em termos de TIB, encontram-se em um estágio que pode ser classificado como competitivo no mercado em que atuam; o último grupo reúne empresas com sistemas de gestão produtiva bem estruturados. Isso demonstra a pertinência do apoio com instrumentos flexíveis, isto é, desenhados com um tipo de atendimento "puxado" pela demanda, seja considerando cada empresa, seja considerando grupos de empresas com necessidades similares.

Os resultados da pesquisa de Gallina (2009) e da pesquisa apresentada neste artigo

7 A evolução histórico-institucional destes sistemas de gestão deriva do processo crescente de exigência de qualidade intrínseca e tem forte correlação com aspectos metrológicos (BRASIL, 2005, p. 27-46). 
corroboram a importância da capacitação em TIB para o desenvolvimento produtivo das MPME do setor metalmecânico analisadas no estado do Rio de Janeiro. Além da correlação positiva entre a formação e a acumulação de competências para o aumento do potencial inovativo das empresas, tem-se uma importante implicação de política para a inovação: o fortalecimento do estímulo à capacitação empresarial em sistemas de formação e acumulação de competências em TIB com base em sistemas de gestão da qualidade, de gestão ambiental e de saúde e segurança no trabalho.

Contudo, outras preocupações surgem quando a capacitação em TIB é considerada a nível nacional. Como assinala Tironi (2017), o crescente movimento de consolidação de grandes certificadoras multinacionais na direção da absorção daquelas de menor porte em diversos países e no Brasil traz como alerta a necessidade de fortalecimento do Inmetro como órgão executor da política na área. As grandes organizações e sociedades certificadoras vêm adquirindo laboratórios de ensaios, análises e certificadoras, por isso é necessário que a política nacional no campo da TIB alcance efetivamente as empresas nacionais e particularmente as MPME em razão da sua maior fragilidade, garantindo o acesso das empresas aos serviços a preços adequados à manutenção da competitividade dessas empresas. Vale lembrar que hoje a competitividade a nível mundial passa mais pela superação de barreiras técnicas do que tarifárias.

Conforme mencionado anteriormente, a TIB tem escopo estratégico por envolver vantagens competitivas, tanto no âmbito macroeconômico, como microeconômico. Como afirma Fleury (2007, p. 40):

\section{"[...] o correto entendimento do alinhamento estratégico entre o Sistema Nacional de TIB e a Política Industrial no plano dos países é o que determina a eficácia das decisões e dos investimentos. O portfolio de alternativas e o campo de decisões sobre TIB é tão vasto que não há possibilidade de haver eficácia se não houver estratégias de ação claramente definidas.".}

O Sibratec nasceu alinhado à Política Industrial, Tecnológica e de Comércio Exterior (PITCE) criada em 2003 e ao PACTI 2007-2010. A Política de Desenvolvimento Produtivo (PDP) surgiu em 2008 e o Plano Brasil Maior em 2011 seguido pela Estratégia Nacional de Ciência, Tecnologia e Inovação (ENCTI) 2012-2015 (BRASIL, 2011). A versão atualizada desta estratégia - a ENCTI 2016-2022 - tem na modernização e ampliação da infraestrutura de C,T\&I uma de suas ações prioritárias para manter o investimento, a expansão e o fortalecimento dos programas de apoio à inovação tecnológica nas empresas (BRASIL, 2016). O programa Sibratec tem sido mantido nas políticas públicas de CT\&I.

Em 2013 o Sibratec contava com 49 redes operacionais, sendo 10 unidades como centros de inovação, 17 redes temáticas de serviços tecnológicos para atividades de calibração, ensaios, análises e certificação e 22 arranjos estaduais voltados para a extensão tecnológica dentre os quais o do Rio de Janeiro aqui analisado (BRASIL, 2013). Mas apesar do ambiente institucional favorável, as atividades de várias redes de serviços tecnológicos e de extensão do Sibratec se encerraram, o que coloca em evidência o tema recorrente do hiato entre concepção, planejamento e implementação de políticas públicas no país, evidenciado também na pesquisa aqui descrita. O Estado afeta diretamente as capacitações e estratégias inovativas das empresas, por isso a coordenação e a integração de políticas públicas são fundamentais em países de grandes dimensões como o Brasil.

Dado o escopo estratégico da TIB, considerando o macroambiente e o microambiente, espera-se que o Sinmetro seja fortalecido e que as ações deste sistema mantenham 
articulação com as atividades do Sibratec-ET para que o apoio às MPME industriais alcance patamares mais significativos em termos regionais e nacionais. Um sinal positivo foi dado com a Emenda Constitucional no 85 que colocou o Estado como protagonista do incentivo ao desenvolvimento científico, à pesquisa, à capacitação científica e tecnológica e à inovação. E ainda, a Lei no 13.243/2016 e o Decreto $n^{\circ}$ 9.283/2018 estabeleceram novos estímulos à inovação e à pesquisa científica e tecnológica no ambiente produtivo. Além disso, outro sinal positivo ocorreu com a criação efetiva do Sistema Nacional de Ciência, Tecnologia e Inovação (SNCTI), a ser organizado em regime de colaboração entre entes públicos e privados com o objetivo de promover as atividades de C,T\&I.

\section{6- Considerações Finais}

Este artigo investigou a capacitação tecnológica em TIB de MPME industriais do setor metalmecânico localizadas na ZO do MRJ e o apoio oferecido pelo Sibratec no âmbito de uma de suas redes regionais de extensão tecnológica - a Sibratec-ET Rede RJ. Embora o estudo tenha contemplado apenas as funções básicas de TIB como normalização e regulamentação técnica, metrologia e avaliação de conformidade envolvendo aspectos de qualidade, estas atividades são especialmente relevantes para as empresas que pretendem melhorar sua capacidade tecnológica e competitividade para atingir patamares superiores de maturidade organizacional e alcançar a fronteira tecnológica internacional, seja em termos de produção, seja de inovação o que requer, nos dois casos, aprendizagem tecnológica.

A intensificação dos fluxos de comércio internacional tem exigido das empresas a conformidade com vários requisitos por meio do atendimento a normas e regulamentos técnicos, de modo que elas possam ter acesso a novos mercados no país e no exterior. Em especial, a avaliação de conformidade por meio da certificação de sistemas produtivos vem se tornando uma barreira técnica às exportações de países em desenvolvimento como o Brasil, por isso é importante apoiar o desenvolvimento da capacitação em TIB de empresas de portes e setores produtivos diversos em todo o território nacional elevando, assim, a capacidade de internacionalização do tecido industrial brasileiro. Contudo, a elevação do padrão tecnológico é difícil para as empresas de base nacional, especialmente para aquelas de menor porte, daí a necessidade de incentivos governamentais a elas dirigidos.

Assim, pretendeu-se avançar na compreensão do potencial dos instrumentos de extensão tecnológica na promoção de ganhos de qualidade e produtividade para as MPME do setor metalmecânico na ZO do MRJ, levando em conta que elas reúnem competências específicas capazes de alavancar encadeamentos entre vários setores produtivos e de contribuir para o desenvolvimento regional e local. Os resultados obtidos indicam que a rede de extensão no Rio de Janeiro possui as modelagens adequadas para atender ao conjunto de demandas das MPME, ou seja, existe aderência entre as demandas identificadas e a oferta de apoio da Sibratec-ET Rede RJ, embora não na medida necessária. Mais do que isso, verificou-se que a estrutura do Sibratec por meio de duas de suas redes - a de serviços tecnológicos ${ }^{8}$ e a de extensão tecnológica aqui analisada - têm aderência às demandas identificadas nas MPME pesquisadas.

Confirmando a literatura, os resultados mostram a fragilidade da maioria das empresas quanto à capacitação em TIB, embora a análise de suas demandas vis-à-vis a oferta de apoio tenha revelado uma correlação positiva, dado o formato flexível do atendimento proposto. Ainda

8 A rede de serviços tecnológicos é de estruturação temática e se destina a apoiar a infraestrutura de serviços de avaliação da conformidade (metrologia, ensaios, análises e certificação), normalização e regulamentação técnica, com a finalidade de auxiliar as empresas na superação de exigências técnicas para o acesso aos mercados interno e externo. 
assim, a Rede Sibratec-ET Rede RJ não logrou êxito em relação a seus objetivos, configurandose mais como um arranjo institucional potencialmente positivo do que efetivo. As empresas pesquisadas sequer souberam da existência da Sibratec-ET ou foram visitadas, o que indica um campo fértil para o debate a partir de uma avaliação de impacto deste arranjo específico em outros setores ou regiões ou mesmo do Sibratec como um todo.

Ratifica-se a necessidade de novas pesquisas sobre esses temas, sobretudo considerando a dimensão nacional do sistema e sua forma de atuação em distintos tipos de redes como centros de inovação, serviços tecnológicos e a de extensão tecnológica aqui abordada. É grande o número de redes estaduais passíveis de serem analisadas, avaliadas e comparadas, gerando conhecimento útil para os campos da economia, das políticas públicas e do planejamento urbano e regional. 


\section{Referências Bibliográficas}

ABREU, J. A. P. Normalização e 20 anos de TIB no Brasil. In: Ministério da Ciência e Tecnologia (MCT) (Org.). Tecnologia industrial básica: trajetória, desafios e tendências no Brasil. Brasília: MCT: CNI: SENAI/DN: IEL/NC, 2005, p. 93-111.

ASSOCIAÇÃO BRASILEIRA DE NORMAS TÉCNICAS (ABNT). Institucional / Normalização / Certificação. Disponível em: <http://www.abnt.org.br>. Acesso em: 20 nov. 2018.

BELL, M. \& PAVITT, K. Technological accumulation and industrial growth: contrasts between developed and developing countries. Industrial and Corporate Change, v. 2, n. 2, p. 157-210, 1993.

BENN, S. New processes of governance: cases for deliberative decision-making? Managerial Law, v. 49, n. 5/6, p. 196-205, 2007.

BHIMANI, A. \& SOONAWALLA, K. From conformance to performance: the corporate responsibilities continuum. Journal of Accounting and Public Policy, v. 24, n. 3, p. 165-174, 2005.

BITTENCOURT, P. F. \& CÁRIO, S. A. F. Sistemas de inovação: das raízes no século XIX à análise global contemporânea. In: RAPINI, M. S.; SILVA, L. A.; ALBUQUERQUE, E. M. (Orgs.). Economia da Ciência, Tecnologia e Inovação: fundamentos teóricos e a economia global. Curitiba: Prismas, 2017, p. 331-369.

BRASIL. Ministério da Ciência, Tecnologia, Inovações e Comunicações. Programa Tecnologia Industrial Básica e serviços tecnológicos para inovação e competitividade. Brasília: MCT, 2001.

BRASIL. Ministério da Ciência, Tecnologia, Inovações e Comunicações. Tecnologia industrial básica: trajetória, desafios e tendências no Brasil. Brasília: MCTI; CNI; SENAI/DN; IEL/NC, 2005.

BRASIL. Ministério da Ciência, Tecnologia, Inovações e Comunicações. Plano de Ação em Ciência, Tecnologia e Inovação para o Desenvolvimento Nacional (PACTI 2007-2010). Brasília: MCTI, 2007.

BRASIL. Ministério da Ciência, Tecnologia, Inovações e Comunicações. Estratégia Nacional de Ciência, Tecnologia e Inovação 2012-2015 (Balanço das Atividades Estruturantes 2011). Brasília: MCTI, 2011.

BRASIL. Ministério da Ciência, Tecnologia, Inovações e Comunicações. Programa Sibratec 2013 [Relatório de Gestão]. Secretaria de Desenvolvimento Tecnológico e Inovação. Brasília: MCTI, 2013.

BRASIL. Ministério da Ciência, Tecnologia, Inovações e Comunicações. Estratégia Nacional de Ciência, Tecnologia e Inovação 2016-2022 (ENCTI 2016-2022). Brasília: MCTIC, 2016.

BRITTO, J. Cooperação para a inovação. In: RAPINI, M. S.; SILVA, L. A.; ALBUQUERQUE, E. M. (Orgs.). Economia da Ciência, Tecnologia e Inovação: fundamentos teóricos e a economia global. Curitiba: Prismas, 2017, pp. 287-330.

COHEN W. M. \& LEVINTHAL D. A. Innovating and learning: the two faces of R\&D. Economic Journal, v. 99, September, p. 569-596, 1989.

COHEN W. M. \& LEVINTHAL D. A. Absorptive capacity: a new perspective on learning and innovation. Administrative Science Quarterly, v. 35, n. 1, p. 128-152, 1990.

CRESPO, W. B. \& SOUZA, C.G. O papel do INPI no processo de difusão tecnológica: avaliação do PROFINT (Programa de Fornecimento Automático de Informação Tecnológica). In: ENCONTRO 
NACIONAL DE ENGENHARIA DE PRODUÇÃO, XXVI - ENEGEP 2006. Anais... Fortaleza: ABEPRO, 2006, p. 1-9.

DARÓS, M. M. O Programa Brasileiro de Qualidade e Produtividade: uma análise de política. Dissertação (Mestrado em Política Científica e Tecnológica). Instituto de Geociências, Universidade Estadual de Campinas (Unicamp), Campinas, SP, 1997.

DIAS, J. L. Mercados medidos: a construção da tecnologia industrial básica no Brasil. Rio de Janeiro: Redetec, 2007.

FIGUEIREDO, P. N. Gestão da inovação: conceitos, métricas e experiências de empresas no Brasil. Rio de Janeiro: LTC, 2012.

FLEURY, A. A Tecnologia Industrial Básica (TIB) como condicionante do desenvolvimento industrial na América Latina. [Ciencia y Tecnología para el Desarollo]. Comissão Econômica para a América Latina e o Caribe (CEPAL). Santiago do Chile: Chile, 2007.

GALLINA, R. A contribuição da Tecnologia Industrial Básica (TIB) no processo de formação e acumulação das capacidades tecnológicas de empresas do setor metalmecânico. Tese (Doutorado em Engenharia de Produção). Escola Politécnica, Universidade de São Paulo (USP), São Paulo, SP, 2009.

GIL, A. C. Métodos e técnicas de pesquisa social. 6 ed. São Paulo: Atlas, 2011.

GONZÁLEZ, C. G. \& VÁZQUEZ, J. R. Z. Metrología. D.F., México: McGraw-Hill, 1996.

HASENCLEVER, L. \& CUNHA, E. O pólo metal-mecânico: uma demanda para dinamização e modernização das empresas da zona oeste. In: LA ROVERE, R. L. \& SILVA, M. O. (Orgs.). Desenvolvimento econômico local da Zona Oeste do Rio de Janeiro e seu entorno. Rio de Janeiro: PoD, 2010, p. 73-87.

INSTITUTO NACIONAL DE METROLOGIA, QUALIDADE E TECNOLOGIA (INMETRO). Conheça o Inmetro: o que é o Inmetro / Metrologia Científica e Industrial / Definições de Regulamento Técnico, Norma e Procedimento de Avaliação da Conformidade. / Qualidade e Avaliação de Conformidade. Disponível em: <http://www.inmetro.gov.br>. Acesso em: 01 dez. 2018.

INSTITUTO NACIONAL DE TECNOLOGIA (INT). Implantação da Rede de Extensão Tecnológica do Rio de Janeiro para apoio às Micro, Pequenas e Médias Empresas. [Relatório Técnico Gerencial Final]. Rio de Janeiro: INT, 2016.

LA ROVERE, R. L.; HASENCLEVER, L. \& PINTO, J. P. M. An introduction to small and medium-sized enterprises (SMEs) in Brazil. In: HERNANDEZ, N. \& RAMIREZ, J. (Org.). MEs unleashing the potential: opportunities and challenges: the cases of Brazil, Mexico, France, Germany, Italy and Spain. New Delhi: Bloomsbury, p. 16-41, 2014.

MADEIRA, P. Extensão industrial em sistemas locais de produção: os efeitos da aglomeração na aquisição e difusão de novas capacitações em PME. Dissertação (Mestrado em Engenharia de Produção). Escola Politécnica, Universidade de São Paulo (USP), São Paulo, SP, 2009.

MENDES, H. S. Extensão tecnológica no Brasil: uma análise da oferta e da demanda das empresas de metalmecânica da zona oeste do município do Rio de Janeiro. Tese (Doutorado em Políticas Públicas, Estratégias e Desenvolvimento). Instituto de Economia, Universidade Federal do Rio de Janeiro (UFRJ), Rio de Janeiro, RJ, 2016.

MENDES, H. S. \& HASENCLEVER, L. Development, entrepreneurial activity and industrial extension. In: LA ROVERE, R. L.; OZÓRIO, L. M.; MELO, L. J. (Orgs.). Entrepreneurship in BRICS: policy and research to support entrepreneurs. Cham: Springer International Publishing, 2015, p. 239-253. 
MENDES, H. S.; FERREIRA, M. L. A.; HASENCLEVER, L. \& TEIXEIRA, C. A. M. Extensão tecnológica e desenvolvimento regional: o caso Sibratec-ET Rede RJ. In: ENCONTRO NACIONAL DE ECONOMIA INDUSTRIAL E INOVAÇÃO, II - ENEI 2017. Anais... Rio de Janeiro, Blucher Engineering Proceedings. São Paulo: Editora Blucher, 2017. v. 4. p. 792-811.

OLIVEIRA, J. E. F. A metrologia aplicada aos setores industrial e de serviços: principais aspectos a serem compreendidos e praticados no ambiente organizacional. Brasília: Sebrae, 2008.

OLIVEIRA, S. T. Tecnologias de apoio à competitividade: programas e benefícios para as micro e pequenas empresas. Rio de Janeiro: Redetec, 2011.

OLIVEIRA, M. C. Tecnologia Industrial Básica na formação em engenharia. Dissertação. (Mestrado em Tecnologia). Diretoria de Pesquisa e Pós-Graduação, Centro Federal de Educação Tecnológica Celso Suckow da Fonseca (CEFET/RJ), Rio de Janeiro, RJ, 2015.

OLIVEIRA, M. C. \& SOUZA, C. G. Formação em Tecnologia Industrial Básica (TIB): uma experiência com alunos de graduação em engenharia. In: SIMPÓSIO DE EXCELÊNCIA EM GESTÃO E TECNOLOGIA, XI - SEGET 2014. Anais... Resende: AEDB, 2014, p. 1-15.

ORGANIZAÇÃO MUNDIAL DE PROPRIEDADE INTELECTUAL (OMPI) / INSTITUTO NACIONAL DE PROPRIEDADE INDUSTRIAL (INPI). Introdução à Propriedade Intelectual. [Curso Geral à Distância - DL101P BR - Módulo 2 - 3V]. Rio de Janeiro: INPI, 2018.

PENROSE, E. A teoria do crescimento da firma. Campinas: Unicamp, 2006.

SISTEMA BRASILEIRO DE TECNOLOGIA (SIBRATEC). Sobre o Programa / Governança do Sibratec. Disponível em: <https://sibratec.mctic.gov.br/sibratec>. Acesso em: 05 dez. 2018.

SHAPIRA, P. Modernizing manufacturing: new policies to build industrial extension services. Washington D. C.: Economic Policy Institute, 1990.

SOUZA, R. D. F. Tecnologia Industrial Básica como fator de competitividade. Parcerias Estratégicas, n. 8, maio 2000.

TEECE, D. J. Explicating dynamic capabilities: the nature and microfoundations of (sustainable) enterprise performance. Strategic Management Journal, n. 28, v. 13, p. 1319-1350, 2007.

TEECE, D. J.; PISANO, G. \& SHUEN, A. Dynamic capabilities and strategic management. Strategic Management Journal, v. 18, n. 7, p. 509-533, 1997.

TIGRE, P. B. Gestão da inovação: a economia da tecnologia no Brasil. Rio de Janeiro: Elsevier, 2014.

TIRONI, L. F. Serviços tecnológicos nas estatísticas nacionais e na inovação. Radar Tecnologia, Produção e Comércio Exterior. Brasília: IPEA, n. 40, p. 39-49, 2015.

TIRONI, L. F. Serviços tecnológicos e política de inovação. In: TURCHI, L. M. \& MORAIS, J. M. (Orgs.). Políticas de apoio à inovação tecnológica no Brasil: avanços recentes, limitações e propostas de ações. Brasília: IPEA, 2017, p. 433-468.

TUZZO, S. A. \& BRAGA, C. F. O processo de triangulação da pesquisa qualitativa: o metafenômeno como gênese. Revista Pesquisa Qualitativa, v. 4, n. 5, p. 140-158, 2016.

YIN, R. K. Estudo de caso: planejamento e métodos. 4. ed. Porto Alegre: Bookman, 2010. 